\title{
Folding and Stability of Transmembrane $\beta$-Barrels of Bacterial and Human Origin: Probing Underlying Similarities and Principal Differences Using in vitro Systems
}

\author{
RADHAKRISHNAN MAHALAKSHMI* \\ Molecular Biophysics Laboratory, Department of Biological Sciences, Indian Institute of Science \\ Education and Research, Bhopal, India
}

(Received on 16 October 2014; Revised on 04 January 2015; Accepted on 15 January 2015)

\begin{abstract}
The biochemical, biophysical and functional characterization of transmembrane $\beta$-barrels of human and bacterial origin has provided insight on factors that stabilize these proteins in various lipid and detergent systems. This review attempts to summarize our recent findings and contributions to the field of membrane protein biophysics. Using various $\beta$-barrels, particularly, the 8-stranded OmpX and Ail from Escherichia coli and Yersinia pestis, respectively, and the 19-stranded human mitochondrial transmembrane barrel VDAC-2 (the voltage-dependent anion channel isoform 2) as model systems, we have observed that barrel-micelle interaction strengths, protein-to-detergent ratios and interface residues serve as key elements in determining the stability of these barrel systems, irrespective of the protein origin. Differences in the behaviour of these barrels arise from their malleability to reversible folding versus stabilization by kinetic contributions, with the latter giving rise to irreversible unfolding and aggregation. Furthermore, while bacterial proteins are more tolerant to mutations, hVDAC-2 is particularly influenced by subtle (conserved) mutations, both in its stability and function. The implications of these findings to the field of membrane proteins are also discussed.
\end{abstract}

Key Words: Outer Membrane Protein; VDAC; Membrane Protein Folding; Spectroscopy; Protein-Lipid Interactions; Protein Kinetic Stability

\section{Introduction}

Membrane proteins have served as lucrative targets of study, for both the research as well as the pharmacological worlds, owing to their indispensable role in every key process of the cell. The last decade has witnessed a rapid increase in our knowledge of membrane protein structure, owing to the development of crystallization techniques for these proteins and amenability of these molecules to structural characterization in solution using high-resolution NMR methods. Further, extending the use of mutational studies and $\Phi$ value analysis, used routinely for the characterization of soluble proteins, to the family of membrane proteins, has augmented our understanding of key residues that drive protein folding, and are involved in protein-lipid interaction and stabilization of the refolded protein under native and artificial environments. Lastly, but probably the most importantly, is the introduction of methods that allow researchers to examine the functional features of membrane proteins, both in vivo and in vitro, and identify interacting partners and regulatory proteins; this has permitted us to correlate the structure and dynamics of membrane proteins with their function and regulation.

*Author for Correspondence: E-mail: maha@iiserb.ac.in 
Most of our current understanding of membrane protein behaviour stems from studies carried out on a few selected transmembrane (TM) helices, such as bacteriorhodopsin (Curnow and Booth, 2007; Faham et al., 2004; Parker and Marqusee, 1999), and $\beta$ barrels, such as OmpA and PagP from Escherichia coli (Andersen et al., 2012; Burgess et al., 2008; Hong et al., 2007; Hong et al., 2013; Huysmans et al., 2007; Moon et al., 2013; Sanchez et al., 2011; Shanmugavadivu et al., 2007).While these proteins serve as good representatives to address general membrane protein structure, it is difficult to readily translate these observations to all membrane proteins. The key reason for this is bacteriorhodopsin, OmpA and PagP exhibit thermodynamic stabilization, whereas it is increasingly evident that membrane protein stabilization may arise largely from kinetic contributions (Jefferson et al., 2013). At the molecular level, several unanswered questions remain to be addressed, including residue-wise identification of elements, i.e., delineating the contribution of the protein as well as the chemical and physical nature of the refolding environment. Further, the global contribution of protein-lipid interactions to the structure, function and regulation of membrane proteins requires understanding.

This review aims to summarize the contribution of my laboratory to our current understanding of membrane proteins to the $\beta$-barrel category, using the Attachment-invasion locus (Ail) protein from Yersinia pestis, the outer membrane protein $\mathrm{X}(\mathrm{OmpX})$ from $E$. coli and the human voltage-dependent anion channel isoform 2 (hVDAC-2), and relates our findings to other observations made and conclusions reached using in vitro studies conducted on other membrane protein systems. In particular, this review aims to consolidate our current understanding of factors that influence TM $\beta$-barrel membrane protein folding as well as unfolding, and elements that contribute to the overall structure and stability of the refolded protein in its lipid or detergent environment. Finally, the review ends by recapitulating key unanswered questions in the field of membrane protein biophysics, structural and functional biology.

\section{Refolding of $\beta$-Barrels in vitro: Lipid or Micelle Dynamicity Can Drive Membrane Protein Folding}

In bacteria, the in vivo refolding process of TM $\beta$ barrels, found particularly in the outer membrane, occurs through the orchestrated interaction of the SecYEG complex and proteins of the Skp complex; the SecYEG complex, in particular, facilitates the transport of the membrane proteins synthesized in the cytosol to the inter-membrane space. These protein complexes together mediate insertion of the unfolded protein into the bacterial outer membrane with the assistance of the BAM complex (Moon et al., 2013) (Fig. 1). It is presumed that refolding of the protein is spontaneously driven by expending the energy gradient present in the outer membrane (Moon et al., 2013). However, in vitro refolding of TM barrel preparations, which are usually in the unfolded form, is driven to the folded state in a multi-step event that is nucleated by the rapid dilution of the denaturant (Fig. 2). It has been demonstrated, using ingeniously designed fluorescence measurements (Kleinschmidt and Tamm, 1996; Kleinschmidt et al., 1999a; Kleinschmidt and Tamm, 1999; Kleinschmidt and Tamm, 2002; Otzen and Oliveberg, 2002; Parker and Marqusee, 1999; Pocanschi et al., 2006a), that the rapid adsorption process of the barrel is followed by a protein insertion event in the artificial lipid system. This barrel insertion is the slowest process in the folding pathway of TM barrels in vitro, and this is primarily due to the efficient packing symmetry of the lipid molecules even in small unilamellar vesicles (SUVs).

$\beta$-barrels exhibit poor refolding efficiency in SUVs and large unilamellar vesicles (LUVs) composed of long chain lipids, due to the lipid packing symmetry in these systems; it has indeed been observed that the presence of packing defects in lipids (achieved, for example, by doping of lipid molecules exhibiting positive curvature with molecules exhibiting negative curvature) drives the folding of membrane proteins (Burgess et al., 2008). Surprisingly, the folding process can also be slow in micellar systems, despite the fact that micelles exhibit greater dynamicity and poorer hydrocarbon packing density compared to that of lipids. Such slow folding rates in both detergent micelles and lipid vesicles promote protein aggregation, 


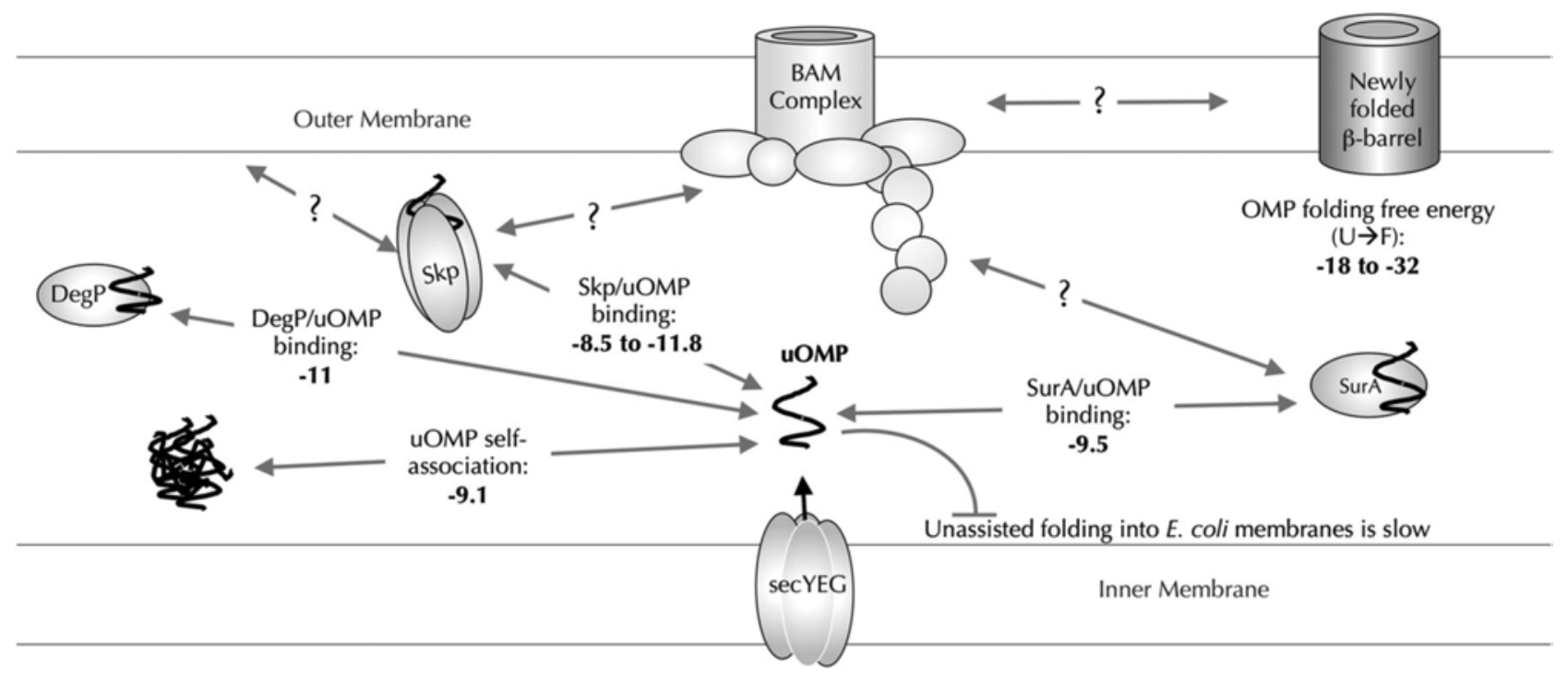

Fig. 1: Proposed mechanism of folding of OMP barrels in the bacterial outer membrane, and the accompanying free energy values involved in these processes. SecYEG: SecY, SecE and SecG components of the inner membrane translocase complex; uOMP: unfolded OMP; DegP, Skp, SurA: chaperones in the periplasm; BAM: $\beta$-barrel assembly machinery, present in the outer membrane; free energy values of each process are indicated in bold, and are in units of $\mathrm{kcal} / \mathrm{mol}$. Figure reproduced with permission from Moon et al., 2013

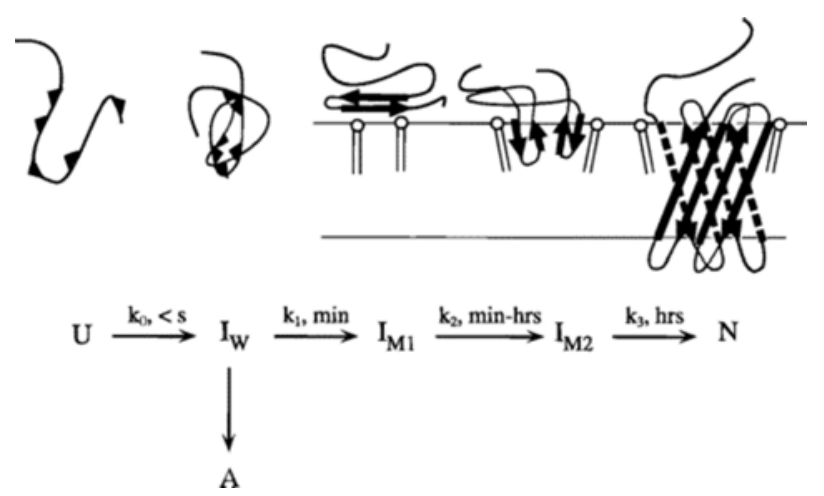

Fig. 2: Rapid collapse of the protein in the unfolded state on the membrane surface (indicated by the initial rates $k_{0}$ and $k_{1}$ ) is followed by a slower barrel folding and assembly event, which can take between minutes and hours to days. $U$ : unfolded protein; $I_{w}$ : water-soluble intermediate; $A$ : aggregated protein; $I_{M 1}, I_{M 2}$ : membrane-bound intermediates; $N$ : native state of $\beta$-barrel. Figure reproduced with permission from Kleinschmidt and Tamm (1996). Copyright (1996) American Chemical Society

dramatically lowering the refolding efficiency of these proteins. Additionally, the persistence of soluble aggregates not only reduces the shelf life of the refolded protein sample, but may also contribute to the results obtained from biophysical and functional assays. We therefore speculated that if the physical properties of the refolding detergent/lipid environment can be altered to introduce 'flaws' in the lipid or greater dynamicity in the detergent, accelerated protein refolding could possibly be achieved. Lipids can be perturbed by physical agents such as temperature and pressure, or chemical factors such as detergents (triton- X -100, sodium dodecyl sulphate) and denaturants (urea, guanidine hydrochloride). In our studies, we used temperature as the perturbant to demonstrate that lipid dynamics can be altered sufficiently enough to allow the $\beta$-barrel membrane protein folding process to be completed, without causing protein aggregation (Maurya et al., 2013) (Fig. 3). Notably, such refolding processes using temperature do not require extensive protein processing after the refolding is completed. The added advantage of this system is reflected in the prolonged shelf life of proteins thus refolded (six-eight months), even when the sample is stored under ambient temperatures.

Several eight-stranded $\beta$-barrels can be refolded using this method (which we describe as 'heat shock' refolding), including OmpA, OmpX, PagP, in both micelles and lipid vesicles (Maurya et al., 2013) (Fig. 


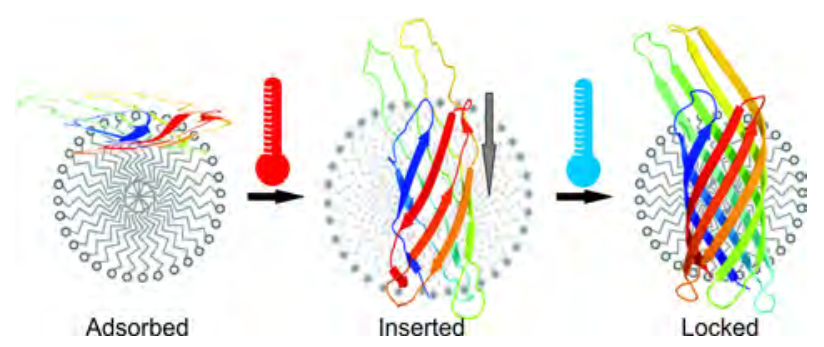

Fig. 3: Heat-shock driven refolding of TM $\beta$-barrels of bacterial origin. Shown here is a representative barrel structure that transitions from the adsorbed (left) to the micelle-inserted state (middle) upon brief exposure to high temperatures $\left(\sim 70{ }^{\circ} \mathrm{C}\right)$. Return of the protein to low temperatures $\left(\sim 4-10{ }^{\circ} \mathrm{C}\right)$ completes the folding process and stabilizes the refolded protein in the locked, micelle-associated state (Maurya et al., 2013). Note that the micelle-adsorbed state is only illustrative, and may not exist experimentally

4). A similar result has been observed when a chemical denaturant such as urea is used as the perturbant in the refolding reaction to drive the folding of the protein PagP (Burgess et al., 2008; Huysmans et al., 2007; Moon et al., 2013). It is observed that proteins amenable to efficient folding can be subjected to detailed biophysical analysis, since the in vitro refolded proteins thus generated are sufficiently stable for the experimental timescales demanded by a variety of biophysical methods and crystallization screens. In addition to the development of effective membrane protein folding methods, it is also vital to understand the factors that retain the refolded protein in a structurally stable form. In addition to signatures in the primary protein sequence and external factors such as $\mathrm{pH}$, temperature, buffer and salt conditions, viscosity etc., that are required to stabilize soluble as well as membrane proteins, the latter are also affected by the choice of the refolding lipid or detergent medium. In the following sections, the implications of protein-lipid/detergent interactions, and role of the chemical nature of both proteins and lipids/detergents, on the overall stability of TM $\beta$-barrels are discussed.

\section{Understanding Factors Stabilizing Membrane Proteins: Membrane Proteins Interact with Micellar Systems}

One of the first considerations for refolding membrane proteins, or maintaining the stability of folded molecules extracted from the cell membrane, is in the use of

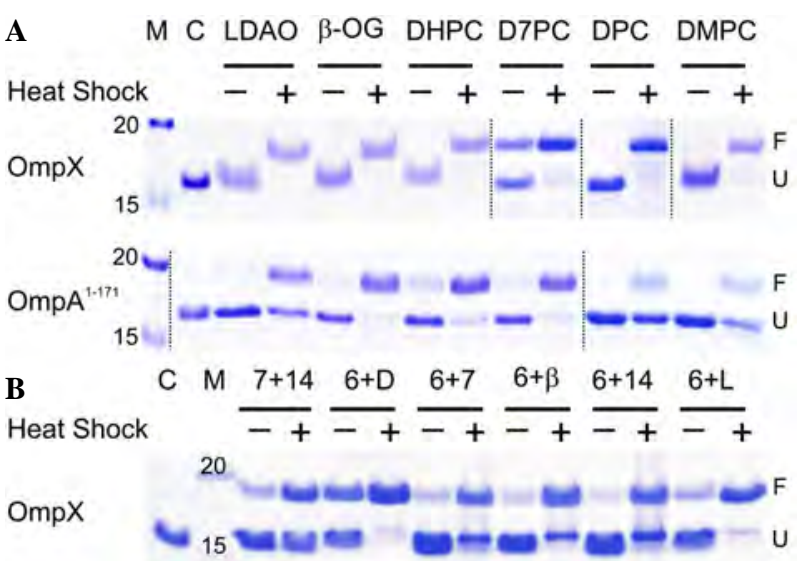

Fig. 4: (A): The use of heat shock method to increase the refolding efficiency of transmembrane $\beta$-barrels in various lipids or detergents is assessed herein, using OmpX and the transmembrane region of OmpA (residues 1-171) as examples. This involves gel mobility shifts carried out on SDS-PAGE gels using unboiled samples. It is observed that in transmembrane $\beta$-barrels, the refolded protein retains structure despite the addition of SDS (in the gel-loading dye), and exhibits a different gel mobility compared to its unfolded counterpart; this is attributed to the ease with which the refolded and unfolded proteins to migrate through the gel pores. Dotted lines separate independent gels that have been presented together for easy comparison. (B) The use of heat shock method to increase the folding efficiency several fold is illustrated herein, in mixed micelles and bicellar systems, using $\operatorname{OmpX}$ as the example. In all gels, the upper band ( 20 kDa) corresponds to the refolded protein and the lower band ( 15 kDa) corresponds to the unfolded protein. $M$ : Marker; $C$ : unfolded control in urea; LDAO: lauryldimethylamine oxide; $\beta$-OG: n-octyl $\beta$-D glucopyranoside; DHPC: 1,2-dihexanoyl-sn-glycero3-phosphocholine; D7PC: 1,2-diheptanoyl-snglycero-3-phosphocholine; DPC: n-dodecylphosphocholine; DMPC: 1,2-dimyristoyl-sn-glycero-3phosphocholine; F: Folded OmpX or OmpA ${ }^{1-171}$; U: Unfolded OmpX or OmpA ${ }^{1-171}$. Figure reproduced with permission from Maurya et al., 2013

non-denaturing detergents and lipids. These lipids and detergents should not only replace the native membrane to provide a homogeneous controlled environment for biophysical and biochemical characterization, but also impart stability to the protein. Many attempted biophysical experiments on membrane proteins necessitate elaborate screens for obtaining optimal conditions of LPR (lipid-to-protein ratio) or DPR (detergent-to-protein ratio), buffer, $\mathrm{pH}$, 
temperature, etc., that provide thermodynamic equilibrium to the system. The choice of the lipid or detergent used in the study can bear implications on the observed results and the interpretations, thereof. This is because membrane proteins are actively involved in interaction with their hydrophobic environment, and their structure and function are influenced by the lateral pressure exerted by the membrane (Grosse et al., 2014). For example, the dynamics of micellar systems is distinct from those of lipid bilayers, with the latter exhibiting greater rigidity (Otzen, 2002; Sehgal et al., 2005). Furthermore, the addition of cholesterol to the bilayer can further reduce membrane fluidity, which, in turn, can alter the lateral pressure exerted by the bilayer system on the foreign protein that it accommodates. For example, in the case of OmpG from E. coli, stretching and flexing deformation of the barrel, observed as a result of bilayer thickness and mismatch, alters porin gating and ion conductance (Grosse et al., 2014).

Unlike bilayers and vesicles, micelles are considered more dynamic entities. However, our studies have revealed that differences in protein behaviour can be observed even in micellar systems. For instance, in the case of the 8-stranded $\beta$-barrel Ail or the 19-stranded $\beta$-barrel hVDAC-2, the proteindetergent complex exhibits hysteresis (discussed later), and different levels of resistance to solvation by chemical denaturants such as guanidine hydrochloride or urea is exhibited by the protein, as the concentration of LDAO ( $n, n$-dimethyldodecylamine $n$-oxide; a 12 -carbon single chain zwitterionic detergent) is changed (Fig. 5) (Gupta et al., 2014; Maurya and Mahalakshmi, 2014b). Surprisingly, when Ail is refolded in DPC ( $n$ dodecylphosphocholine; a 12-carbon single chain zwitterionic detergent) or the hVDAC-2 barrel is refolded in DDM ( $n$-dodecyl- $\beta$-D-maltoside; a 12carbon single chain non-ionic detergent) (both DPC and DDM are similar in carbon chain length and micellar properties as LDAO, but possess different headgroups), both Ail and hVDAC-2 systems exhibit thermodynamic equilibrium (unpublished results). Similarly, a comparison of thermal stability of the 8stranded outer membrane protein X (OmpX (Mahalakshmi et al., 2007; Mahalakshmi and Marassi,
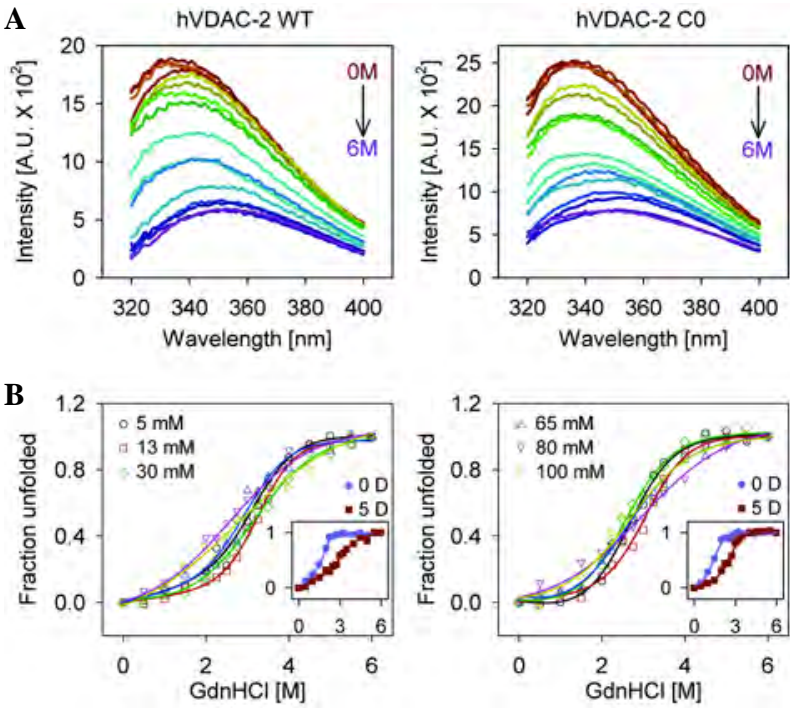

Fig. 5: The effect of detergent concentration on the refolding efficiency of human VDAC-2 (wild type (WT), and the Cys-less mutant Co) monitored using fluorescence measurements. Shown here is the dependence of the change in unfolded fraction with increasing denaturant (guanidine hydrochloride, GdnHCl), on the LDAO concentration (5 mM-100 $\mathrm{mM}$ ) used to refold the protein (B), with a representative fluorescence emission scan for both proteins in (A). Fluorescence data were recorded using an excitation wavelength of $295 \mathrm{~nm}$ for tryptophan, and emission spectra were acquired between 320-400 $\mathrm{nm}$ for the various denaturant concentrations. Fluorescence intensity observed at $340 \mathrm{~nm}$, corresponding to the $\lambda_{\text {em-max }}$ of the refolded protein was used to generate the fraction unfolded plots shown in (B). Also shown as inset in (B), for comparison, are the curves obtained for the aggregated protein $\left(\begin{array}{ll}0 & D\end{array}\right)$ and hVDAC-2 folded in 5 mM LDAO (5 D) using a different refolding method. Figure reproduced with permission from Maurya and Mahalakshmi (2014a)

2008)) from E. coli in LDAO (Chaturvedi and Mahalakshmi, 2013; Maurya et al., 2013) and DPC (Chaturvedi and Mahalakshmi, 2014), shows variations in the reversibility (recovery from thermal denaturation versus irreversible protein aggregation) of the heatmediated denaturation event and chemical unfolding process (unpublished results).

It can be concluded that the behavioural outcome of a membrane protein is determined to a considerable extent by its refolding environment. It is also of interest to examine the factors in the primary sequence and 
secondary structure of a membrane protein that contribute to its final stability in the folded threedimensional state. This is now discussed in the next section.

\section{Thermodynamic Versus Kinetic Contribution to Membrane Protein Stability: The Decision of Whether to Fold or Aggregate}

In biological systems, proteins are stabilized by one of two equilibria: thermodynamic or kinetic (Jefferson et al., 2013; Sanchez-Ruiz, 2010). Thermodynamic stabilization ensures system equilibrium, with complete reversibility in the protein unfolding process, and a return of the unfolded protein to the functional native state through the same folding-unfolding pathway. Under ambient conditions, folding is favoured, and is achieved due to the large negative equilibrium free energy of the folded state (Moon et al., 2011). However, not all proteins are thermodynamically stabilized; increasing evidence points to the role of kinetic stabilization, especially in membrane proteins (Jefferson et al., 2013; Moon et al., 2013). In such systems, the protein is trapped by a kinetic barrier that prevents the molecule from attaining the energetically favourable low energy state. Such systems manifest themselves in the form of 'hysteresis' in denaturation measurements (Andrews et al., 2013). The incidence of hysteresis under in vitro conditions, i.e., refolded membrane protein systems in artificial micellar environments and lipid vesicles, do not necessarily reflect the state of the protein in the native bilayer environment. However, on the basis of the prolonged turnaround time exhibited by several membrane proteins, it is anticipated that the stabilization of these molecules in vivo is also under kinetic control (Curnow and Booth, 2007; Moon et al., 2013).

We have previously examined the factors that contribute to the stability of TM $\beta$-barrels in micellar systems constituted by detergents such as DPC, LDAO, DDM and 6:0-PC (1,2-dihexanoyl-sn-glycero3-phosphocholine). In addition to contributions of the refolding lipid or detergent environment, residue-wise attributes of proteins bearing similar three-dimensional structure also affect the fate of a protein. For instance, in the case of hVDAC-2 and Ail, both proteins exhibit hysteresis in an unfolding process driven by chemical denaturants such as urea or guanidine hydrochloride (Fig. 6). Additionally, in LDAO and DPC detergents, thermal denaturation is irreversible in Ail and hVDAC2 , with both proteins undergoing aggregation upon unfolding (Gupta et al., 2014; Maurya and Mahalakshmi, 2014a). In the micellar conditions examined, these proteins are therefore kinetically stabilized. On the other hand, OmpX and PagP, two structurally similar 8-stranded $\beta$-barrels similar to Ail (Yamashita et al., 2011), exhibit reversibility in chemical and thermal denaturation measurements in micelles, including LDAO and DPC (Chaturvedi and Mahalakshmi, 2013; Chaturvedi and Mahalakshmi, 2014; Maurya et al., 2013). Hence, subtle differences in the amino acid sequence can lead to differences in the secondary structure content, local interactions and affect protein-micelle affinities. These, and other factors such as $\mathrm{pH}$, temperature and DPR (or LPR) can, together, determine height of the activation energy barrier separating the folded and unfolded states of

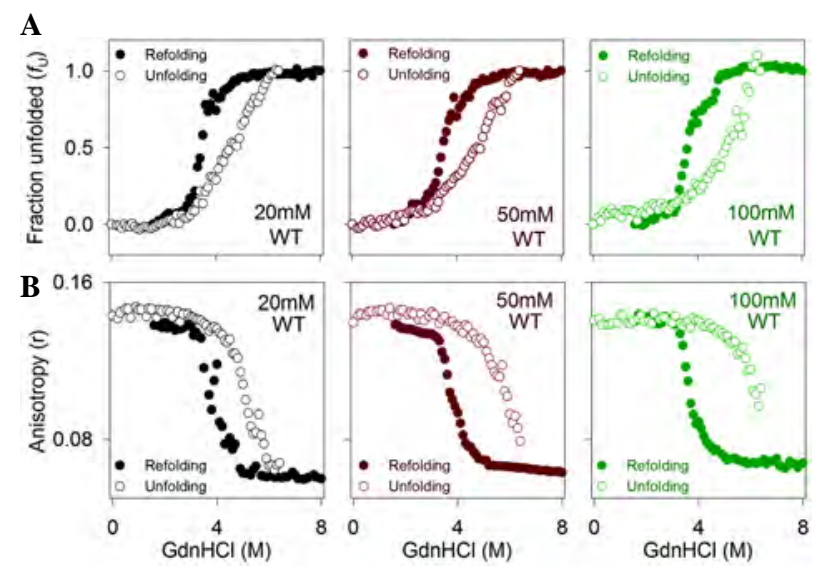

Fig. 6: Refolding (filled symbols) and unfolding (open symbols) profiles of Ail wild type (WT), monitored both using fluorescence emission of tryptophan residues (A): as well as anisotropy of these fluorophores (B): in various LDAO concentrations. The refolding and unfolding pathways are different, indicating the presence of hysteresis in the folding and/or unfolding process of this protein and implying the likelihood of a significant contribution from kinetic stabilization. Note that the unfolding curves shift towards the right (higher GdnHCl) with increase in LDAO concentrations, indicating that the unfolding process is influenced by the micelle amounts in the refolding medium. Figure reproduced with permission from Gupta et al. (2014) 
membrane proteins (Moon et al., 2011).

Mutations can also affect the kinetic contribution to a system under equilibrium. hVDAC-2, for example, has nine cysteine residues. Its more abundant isoform in humans, hVDAC-1, has only two cysteines, implying that the cysteines may play a role in the structure and function of this protein. We have observed that in the case of hVDAC-2, lowering the cysteine content increases the activation energy barrier of protein unfolding (Maurya and Mahalakshmi, 2014a). Similarly, DPR has a pronounced effect on the stability of membrane proteins. Thermal denaturation measurements suggest that hVDAC-2 is more prone to aggregation when the DPR is increased or lowered, as is evident from the unfolding rates of this protein (Fig. 7); particularly, this effect is pronounced in the absence of cysteine residues, with the barrel displaying a sharp difference in the aggregation rates when the DPR lies outside the 2600:1-13000:1 range (Maurya and Mahalakshmi, 2014b). From our experiments, we have reason to believe that the cysteines exist in the reduced form in vitro, as well as in vivo, and are not involved in disulphide bonding (Maurya and Mahalakshmi, 2013). Hence interface residues such as cysteines interact specifically with its surrounding environment and are important to determine the fate of the refolded protein. Similar role of other interface residues has been observed, and is outlined in the next section.

\section{Interface Residues Not Only Determine TM Span But Also Influence Bacterial OMP Folding and Unfolding Pathways}

One of the important factors that determine the stability of any membrane protein is the contacts that it forms with its surrounding hydrophobic environment. The nature of these interactions decides the fate of a membrane protein, including the oligomerization form, stability of the oligomers as well as the prolonged stability of the native protein (Anbazhagan et al., 2008; Booth and Curnow, 2009; Hong and Tamm, 2004; Maurya and Mahalakshmi, 2014b; Moon et al., 2011; Otzen and Andersen, 2013; Shanmugavadivu et al., 2007; Stanley and Fleming, 2008). Of particular interest are the residues of the protein molecule that
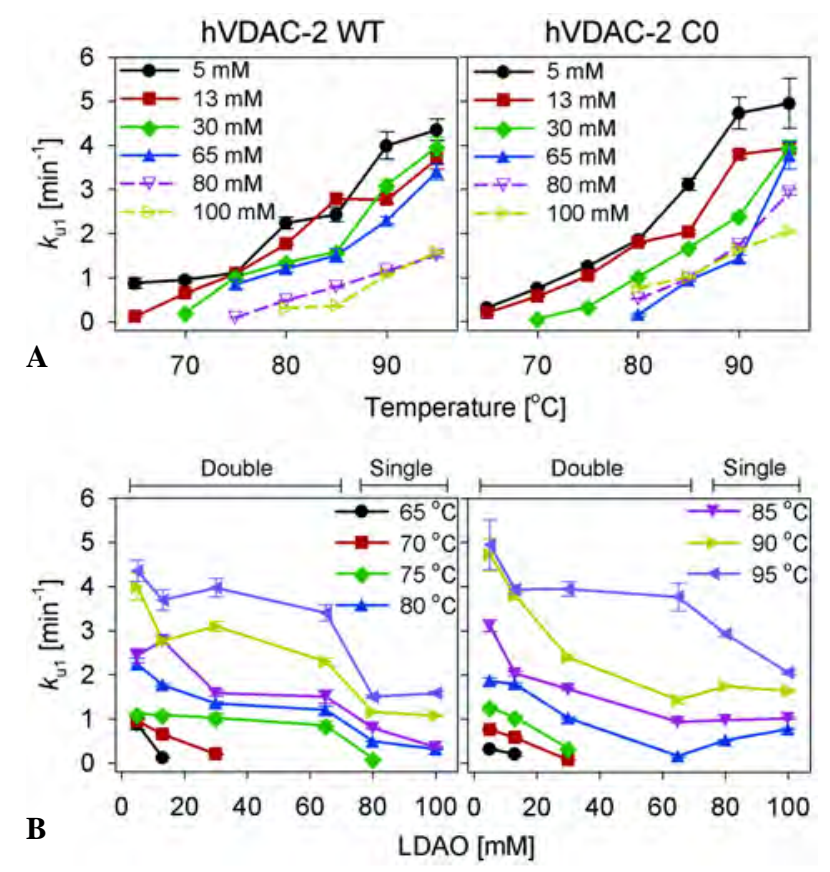

Fig. 7: (A): Unfolding rate of hVDAC-2 WT and C0 monitored at various LDAO concentrations $(5 \mathrm{mM}-100 \mathrm{mM})$ with increasing temperature. At lower LDAO concentrations, the unfolding process is rapid, and can be fit to a double exponential function, providing us with two unfolding rates $\left(k_{\mathrm{u} 1}\right.$ and $\left.k_{\mathrm{u} 2}\right)$. The first rate exhibits a linear increase with increasing temperature. In $80 \mathrm{mM}$ and $100 \mathrm{mM}$ LDAO, aggregation is slower, providing an apparent slow unfolding profile that fits well to a single exponential function (giving a single rate $k_{\mathrm{u} 1}$ ). This is shown as dashed lines. (B) The data from (A) is plotted to highlight the dependence of the unfolding rates on increasing LDAO concentration. $k_{\mathrm{u} 1}$ from the double and single exponential fits (marked as double and single, respectively) are shown for hVDAC-2 WT (left) and hVDAC-2 C0 (right). At higher temperatures, note that the unfolding rate for hVDAC-2 $\mathrm{CO}$ is marginally higher than hVDAC-2 WT. Figure reproduced with permission from Maurya and Mahalakshmi (2014b)

reside at the interface of the hydrophobic lipid/ detergent core and the hydrated polar headgroups and solvent molecules. These residues determine the TM domain span of a membrane protein and define the barrel boundary. Such amino acids that are strategically positioned in the folded protein at the membrane-solvent interface are amphipathic in nature, which allows them to be accommodated at the interface of immiscible environments (Chaturvedi and 
Mahalakshmi, 2013; Faham et al., 2004; Fleming et al., 1997; Gupta et al., 2014; Jefferson et al., 2013; Moon and Fleming, 2011; Rasmussen et al., 2007). Aromatic amino acids, specifically tryptophan, usually populate the interface region in membrane proteins (Andersen et al., 2012; Chattopadhyay et al., 1997; Hong et al., 2007; Hong et al., 2013; Jefferson et al., 2013; Kelkar and Chattopadhyay, 2006; Killian and von Heijne, 2000; Kleinschmidt, 2006; Rasmussen et al., 2007; Sanchez et al., 2008; Sanchez et al., 2011; Sun et al., 2008), and are therefore aptly considered as anchoring residues for the refolded membrane protein.

While it is well-known that interface residues are important determinants of the folded protein stability (Hong et al., 2007), their role in the unfolding or refolding processes of a membrane protein is not explicitly established. We have therefore examined the contribution of the two tryptophan residues to the refolding of the 8-stranded TM $\beta$-barrel Ail (Gupta et al., 2014; Plesniak et al., 2011). Chemical denaturation measurements and thermal melting studies (not shown) together suggest that in this protein, the indole rings not only contribute to the stability of the refolded protein, but also influence the folding process. For instance, when the interface $\operatorname{Trp} 42$ is mutated by a conserved substitution to Phe, a kinetically trapped folding intermediate is observable. This intermediate is, however, absent in the unfolding process, suggesting that the presence of Trp 42 is important during the folding event of the barrel. The second tryptophan, Trp 149, on the other hand, is important for the stability of the refolded protein, and serves to anchor the barrel to its micellar environment. The increased resistance of the refolded Ail barrel to solvation-driven unfolding by chemical denaturants such as $\mathrm{GdnHCl}$ is evidence for the role of this indole in promoting strong proteinmicelle interactions (Gupta et al., 2014) (Fig. 8). A similar conclusion on the unique contributions of Trp to the folding of OmpX has also been made (Chaturvedi and Mahalakshmi, 2014).

In addition to aromatic amino acids, aliphatic residues can also play a subtle, yet significant role, in the folding pathway of membrane proteins. Using OmpX as the example, we have probed the

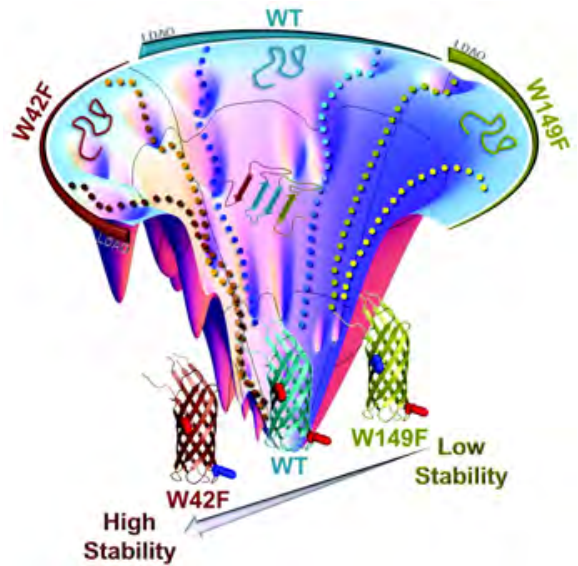

Fig. 8: The influence of DPR and tryptophan mutation(s) on the refolding pathway and stability of the refolded protein, shown here using Ail as the example. Note how the refolding intermediates become more prominent in both high LDAO concentrations as well as when Trp42 is mutated in Ail-WT (left; brown). Figure reproduced with permission from Gupta et al. (2014)

contribution of methionine residues to barrel refolding (Chaturvedi and Mahalakshmi, 2013). Substitution of Met $\rightarrow$ Leu does not affect the structure of the folded OmpX barrel. This is not surprising, since the three Met residues of $\mathrm{OmpX}$ are located in the juxtamembrane loop regions. However, an intriguing observation in this protein was the contribution of the conserved Met $\rightarrow$ Leu mutation on the barrel folding pathway. Our studies using this mutant reveal that the otherwise stable OmpX barrel undergoes a lowering of the unfolding free energy by $\sim 8.5 \mathrm{~kJ} / \mathrm{mol}$ as a consequence of the conserved substitution of three Met residues to Leu. Further, our studies also revealed that the Met-less barrel displays a detectable unfolding intermediate, which is a deviation from the cooperative two-state unfolding exhibited by the parent protein (Fig. 9). Coupled with thermal denaturation measurements, our work using OmpX indicated that subtle, yet conserved, substitutions even in juxtamembrane regions of TM barrels can result in protein destabilization.

Interface residues, therefore, play a very important role in determining the span of the TM domain in $\beta$-barrel membrane proteins, assist the refolding process (membrane insertion), and serve to 


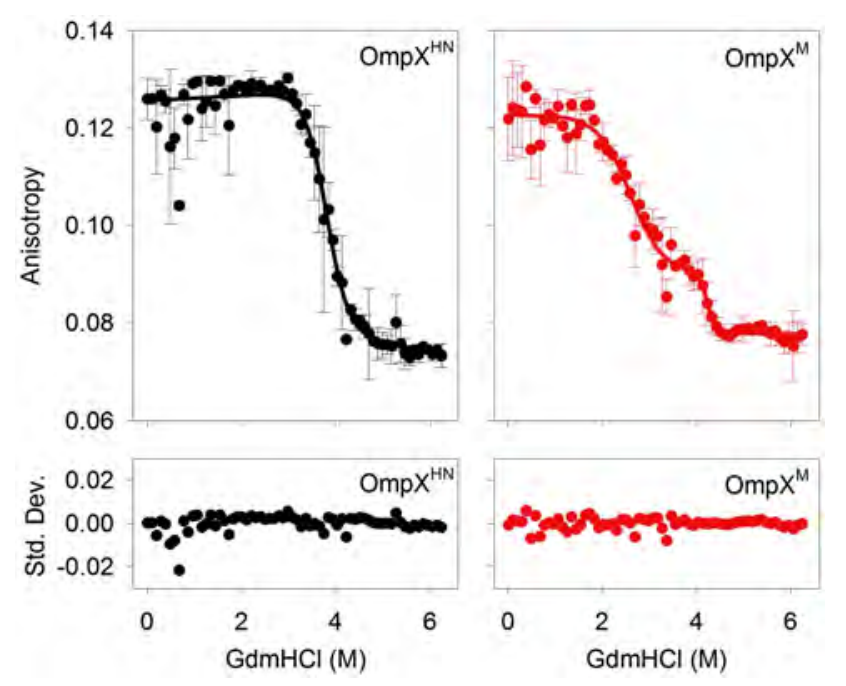

Fig. 9: Anisotropy measurements of $\mathrm{OmpX}$ with increasing guanidine hydrochloride concentration clearly reveal the presence of an intermediate in the pathway in the construct that lacks methionine residues $\left(\mathrm{OmpX}^{\mathrm{M}}\right.$; OmpX H100N; M18L; M21L; M118L) (right, red), whereas the protein containing the native Met residues $\left(\mathrm{OmpX} \mathrm{HN}^{\mathrm{HN}}\right.$; the $\mathrm{H100N}$ mutant of $\mathrm{OmpX}$ ) (left, black) shows a clear two-state transition. Plots of the residuals are shown below each graph, to assess the appropriateness of each fit. Figure reproduced with permission from Chaturvedi and Mahalakshmimi, 2013

anchor the refolded barrel to its surrounding lipid or detergent molecules. The contribution of interface residues to the structure of the barrel can also bear implications on the function or activity of membrane proteins. We now explore the role of interface residues in membrane protein functioning as a channel, using hVDAC-2 as our model example.

\section{Interface Cysteines in the Human Porin VDAC-2 Bear a Structural and Functional Role}

Interface residues have an important role in determining the folding and stability of refolded membrane proteins, as outlined in the previous sections. In hVDAC-2, we were particularly intrigued by the unusual abundance of cysteine residues. hVDAC-2 has nine cysteines, as opposed to two cysteines in the more abundant isoform 1 , as noted in the previous section; these cysteines specifically map to the loop regions facing the intermembrane space of the mitochondrion, in the 19-stranded barrel structure (Maurya and Mahalakshmi, 2013). In refolded hVDAC-2, we observe from mass spectrometric studies that the cysteine residues exist predominantly in their reduced form (Maurya and Mahalakshmi, 2013); hence do cysteines have a structural role in isoform 2 of VDAC? Further, how does the abundance of cysteines affect VDAC function as an anion channel? The role of cysteines in the structural and scaffold rigidity of hVDAC-2 has been addressed using two approaches: the unfolding kinetics and equilibrium unfolding measurements (Maurya and Mahalakshmi, 2014a; Maurya and Mahalakshmi, 2014b). Using a Cys-less mutant of hVDAC-2, wherein each cysteine residue underwent a conserved substitution to the corresponding amino acid present in hVDAC-1 or hVDAC-3 (Fig. 10) (Maurya and Mahalakshmi, 2013), we have examined the kinetics of the unfolding process using thermal denaturation. In both proteins (hVDAC-2 WT and hVDAC-2 C0), we observe that unfolding and aggregation processes are sequential and irreversible. Furthermore, in the absence of cysteines, the activation energy for the unfolding and aggregation event of this 19 -stranded $\beta$-barrel is increased from $\sim 17.0 \mathrm{kcal} / \mathrm{mol}$ by $\sim 6.0 \mathrm{kcal} / \mathrm{mol}$ (Maurya and Mahalakshmi, 2014b). Not surprisingly, hVDAC-2 $\mathrm{C} 0$ also exhibits an increase in the thermal denaturation mid-point $\left(T_{m}\right)$, compared with the WT protein. Increased stabilization of the Cys-less barrel is likely to arise from an increase in barrel rigidity, which could, in turn, be the result of ordering of

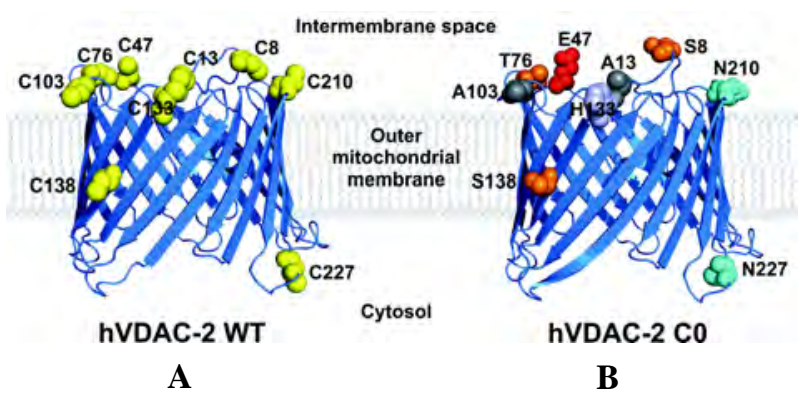

Fig. 10: Ribbon diagram of hVDAC-2 WT (A) and the Cysless mutant $(\mathrm{CO}$; B). The position of cysteine residues in WT are highlighted as yellow spheres and the corresponding substitutions carried out to generate $\mathrm{CO}$ are highlighted as coloured spheres on the ribbon diagram of $\mathrm{CO}$ on the right. Note how, in hVDAC-2, most of the cysteines are positioned towards the intermembrane space. Figure reproduced with permission from Maurya and Mahalakshmi (2014b) 
interface residues when cysteines are mutated. Our current knowledge of hVDAC-2 biophysical properties is limited to data from micellar systems, and we can only speculate that the differences in thermal stability could arise from structural rigidity of the Cys-less mutant.

Our biophysical experiments on hVDAC-2, using chemical denaturation measurements and functional assays using ion conductance across planar lipid bilayers have revealed that in this protein, subtle local variations in barrel interaction with LDAO micelles can bear influence on the measured experimental values (Maurya and Mahalakshmi, 2014a). For example, the Cys-less channel attains greater rigidity through stronger intra-protein interactions compared to the WT hVDAC-2. A consequence of this is the increased barrel stability as described earlier, but also lowered barrel interaction efficacy with its micellar environment. We gauge the lowering of protein-detergent interaction strength by the susceptibility of the refolded protein to solvation by chemical denaturants such as $\mathrm{GdnHCl}$ or urea. For example, as illustrated in Fig. 11, in low LDAO concentrations $(13.0 \mathrm{mM})$, urea considerably lowers the $T_{m}$ of hVDAC-2 C0, by further disrupting the weak $\mathrm{C} 0$ barrel-LDAO interactions, while the WT protein is unaffected. At sufficiently high urea concentrations $(\sim 4.0 \mathrm{M}), \mathrm{C} 0$ displays an $\sim 50 \%$ reduction in the secondary structure content even before the protein is subjected to thermal denaturation, whereas the WT is reasonably unaffected even at $\sim 6.0 \mathrm{M}$ urea. Unlike the anticipated linear dependence of the $T_{m}$ to increasing urea, $\mathrm{C} 0$ in $13.0 \mathrm{mM}$ LDAO therefore displays non-linearity (Fig. 11B). It is likely that beyond 3.5 M urea, the $T_{m}$ we observe is for the structured barrel core, which resists solvation by urea. Surprisingly, however, we observed that the lowered interaction affinity of the barrel to its refolding environment serendipitously increased the signal-tonoise in the electrophysiology measurements (Fig. 12), indicating that the secondary structure of the protein could not only influence the biophysical properties of the protein but also its conductance.

Hence, the mutation of cysteine residues of hVDAC-2 results in altered biophysical, structural and functional characteristics (Maurya and Mahalakshmi, 2013; Maurya and Mahalakshmi, 2014a; Maurya and Mahalakshmi, 2014b). We believe that our observations may have implications in the role of the nine cysteine residues of hVDAC-2 in the regulation of excess ROS in cells, in which irreversible oxidation of cysteines in this protein may be important deciding factors of the cell's fate.

\section{Re-Visiting the DPR Effect on Barrel Folding Pathways}

Thus far, we have examined the role of proteinmicelle/bilayer interaction and signature sequence and structural features in the TM $\beta$-barrels, which determine the observed behaviour of membrane proteins. We have previously discussed that the lipid or detergent chemical composition (changes in headgroup or chain length) can affect membrane
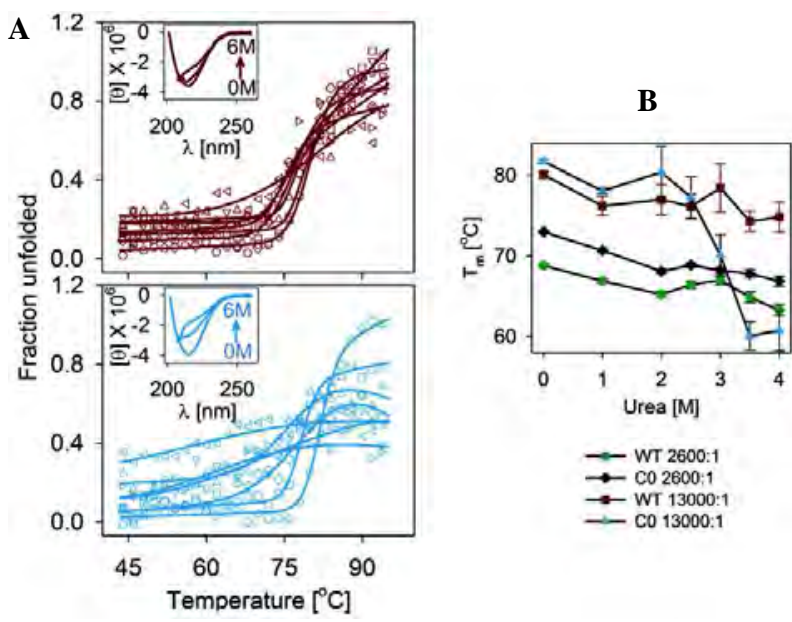

Fig. 11: (A): Thermal denaturation profiles of hVDAC-2 WT (top, brown) and hVDAC-2 $\mathrm{CO}$ (bottom, blue) refolded in LDAO micelles, and monitored at $215 \mathrm{~nm}$, after incubation in different concentrations of urea for $1 \mathrm{~h}$ at $25^{\circ} \mathrm{C}$ (urea concentrations: $\bigcirc: 0.0 \mathrm{M} ; \square$ : $1.0 \mathrm{M}$; $\diamond$ : 2.0 M; $\Delta: 2.5 \mathrm{M} ; \nabla: 3.0 \mathrm{M}$; $\triangleright: 3.5 \mathrm{M} ; \triangleleft: 4.0 \mathrm{M})$. Solid lines represent fits to a two-state equation and the mid-point of thermal denaturation $\left(T_{m}\right)$ thus determined, is plotted in (B). hVDAC-2 $\mathrm{CO}$ shows a considerable lowering of $T_{m}$ upon addition of urea, specifically at the DPR of 13000:1. This arises as a result of destabilization of the secondary structure of hVDAC-2 $\mathrm{CO}$ in high LDAO concentrations, which is magnified further by the presence of urea (see farUV CD wavelength scans presented in the inset to figure (A)). Other DPRs and the WT protein do not show this abrupt $T_{m}$ change. Figure modified with permission from Maurya and Mahalakshmi (2013) 


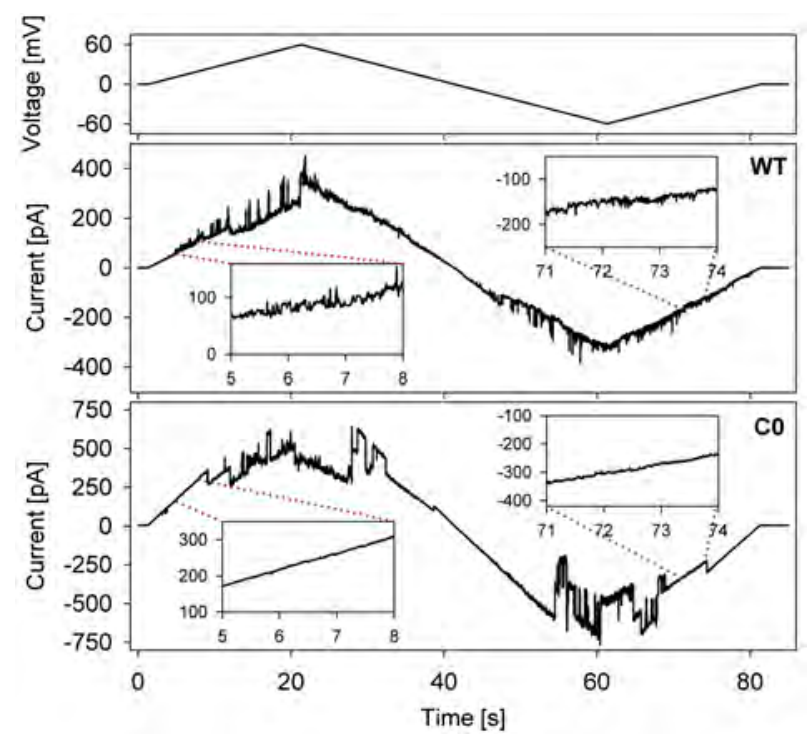

Fig. 12: Representative traces of voltage ramps highlighting channel activity at lower applied voltages $(0 \mathrm{mV}$ to $\pm 30 \mathrm{mV}$ ) and transition of the protein to a closed channel (described as the subconductance state) at higher voltages $(> \pm 30 \mathrm{mV})$. Notice how the noise levels are different in both proteins (highlighted using the insets in both datasets), with the WT protein (middle panel) displaying noisier baselines compared to the Co mutant (lower panel), under similar conditions. Figure reproduced with permission from Maurya and Mahalakshmi (2014a)

protein properties. This is because, unlike soluble proteins, membrane proteins are stabilized by the environment that they are refolded into. Lipids and micelles therefore act as crucial determinants of the stability of membrane proteins, and highly influence the folding rates and folding pathways of these molecules. Reports that have investigated the role of the refolding environment for several TM proteins of bacterial origin clearly indicate a strong influence of the lipid chain length on the refolding efficiency, folding time or stability of the refolded protein (Andersen et al., 2012; Burgess et al., 2008; Kleinschmidt et al., 1999b; Moon et al., 2011; Otzen and Andersen, 2013; Pocanschi et al., 2006b; Pocanschi et al., 2013; Shanmugavadivu et al., 2007; Stanley and Fleming, 2008). In our studies, we have observed a similar dependence of the DPR on the refolding pathway and stability of the folded protein (Gupta et al., 2014; Maurya and Mahalakshmi, 2014a; Maurya and Mahalakshmi, 2014b). Indeed, we have observed that
DPR can also affect the structural properties of singlepass TM synthetic peptides, and drive a conformational switch in these systems (Fig. 13) (Lella and Mahalakshmi, 2013). In this section, we summarize our recent findings on the effect of DPR on the folding and unfolding pathways using $Y$. pestis Ail as the example, and relationship of the stability of the folded barrel to the DPR using hVDAC-2.

Unlike vesicles, micelles are dynamic systems that demonstrate loose packing interactions among the detergent molecules, compared to the long chain lipids. However, detergent association strength in micelles does have a substantial bearing on its ability to accommodate a foreign protein that is refolded into these spherical entities. Furthermore, differences in the headgroup can also influence membrane protein stability, due to variations in micelle packing efficiency and aggregation number. In the case of Ail (Gupta et

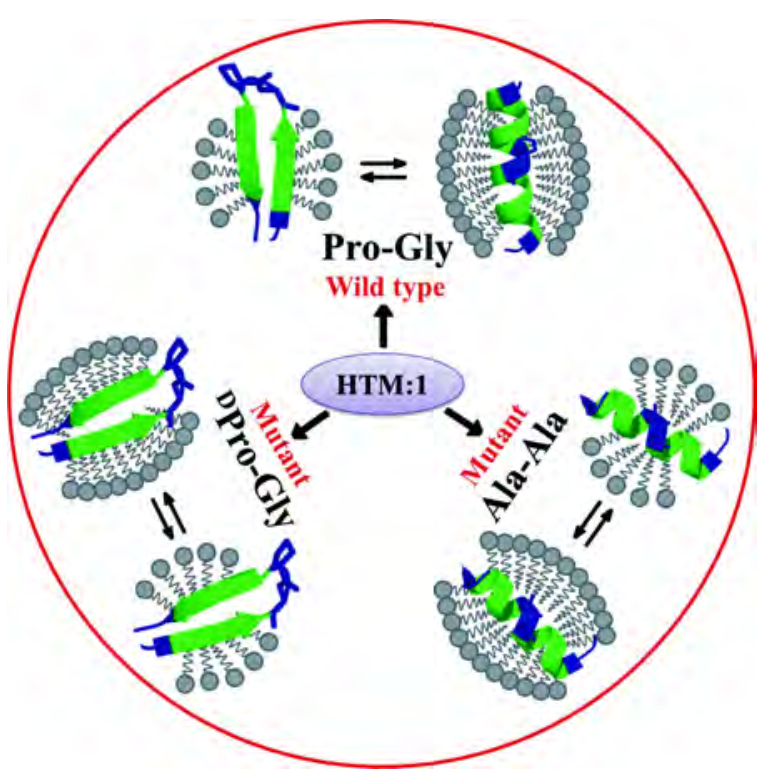

Fig. 13: Conformational interconversion between a $\boldsymbol{\beta}$-hairpin and a helical structure observed in the first transmembrane helix of holin in micellar systems (LDAO and DPC), driven by the central Pro-Gly segment and increase in DPR (represented by the number of grey detergent molecules constituting each micelle). This conformational conversion is abolished when the proline ('Pro) is mutated to ${ }^{D}$ Pro, wherein the peptide retains a $\beta$-hairpin conformation, or is mutated to Ala, in which case the peptide retains a helical conformation. Reproduced from Lella and Mahalakshmi (2013) with permission from The Royal Society of Chemistry 
al., 2014), with the aid of single-Trp mutants, we have observed that the refolding efficiency of the protein is dependent on the DPR, with higher DPRs showing the pronounced stabilization of refolding intermediates that can be trapped spectroscopically. Further, the unfolding process of the protein is obstructed in conditions when there is a micelle overload; i.e., high DPR values stabilize the folded barrel form of Ail. Such acute DPR-dependence cannot arise solely from a change in the micelle size with increasing detergent concentration (Fig. 14). While the micelle aggregation number and CMC is expected to change with salt and buffer conditions, as well as influenced by the presence of a refolded protein, this does not completely explain the observed dramatic differences with linear changes in the DPR. It is likely that the presence of excess empty micelles in high DPRs could mimic macromolecular crowding observed in vivo, thereby altering the activation energy for protein unfolding (Gupta et al., 2014). Different values of protein stability can therefore be obtained, under the influence of the specific protein, and detergent micelle being employed.

While the increase in protein stability with DPR is anticipated, this is not universal. For example, in
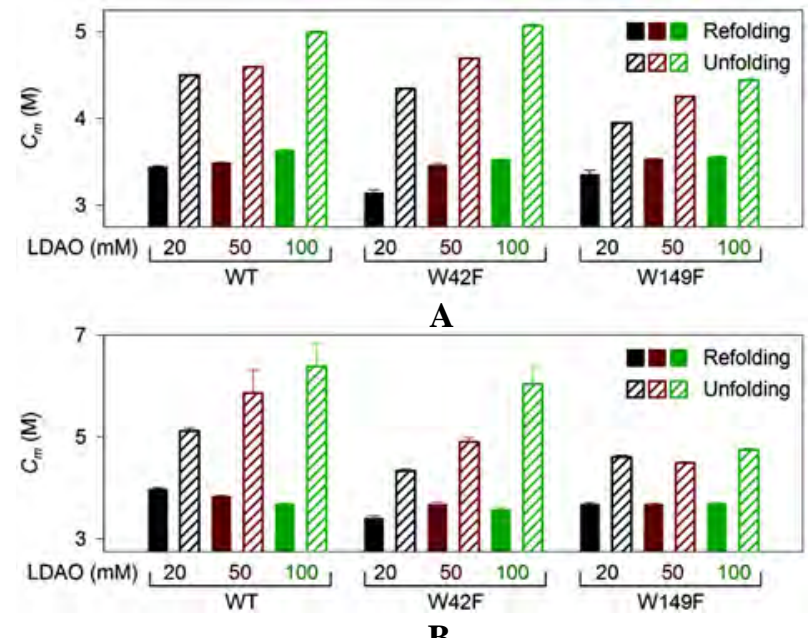

Fig. 14: Comparison of the $C_{m}$ values (mid-point of chemical denaturation) derived using fluorescence measurements (A) and anisotropy (B) for the refolding (filled pattern) and unfolding (hashed pattern) of Ail and its single-Trp mutants in three concentrations of LDAO. Note how the unfolding $C_{m}$ is heavily dependent on the LDAO concentration used in the experiment. Figure reproduced with permission from Gupta et al. (2014) the case of hVDAC-2, the stability of the refolded protein shows a dramatic non-linear dependence to changes in LPR from 2600:1 to 20000:1, as well as the absolute LDAO concentration (for example, in both $5 \mathrm{mM}$ and $13 \mathrm{mM}$ LDAO samples prepared with a DPR of 2600:1, hVDAC-2 shows greater stability in $13 \mathrm{mM} \mathrm{LDAO}$ ) (Maurya and Mahalakshmi, 2014b). At higher and lower DPRs, the hVDAC-2 barrel shows destabilization, resulting in protein unfolding and aggregation (illustrated in Fig. 15). Further, this is also influenced by the presence of cysteine residues in the protein (Maurya and Mahalakshmi, 2014b). Our analysis comparing the activation energy derived from the irreversible unfolding measurements of the wild-type barrel and its Cys-less mutant reveal that cysteines also play a role in determining barrel-micelle interaction strengths. This is achieved by the observed changes in barrel rigidity upon replacement of cysteines. An increase in the barrel malleability in the presence of cysteine residues allows the protein to interact with greater efficiency with its micellar environment. This, in turn, alters the DPR-dependence of the refolded barrel. Nevertheless, our observation that both WT-hVDAC2 and the Cys-less mutant display lowered stabilities at low and high DPRs indicate that this 19-stranded

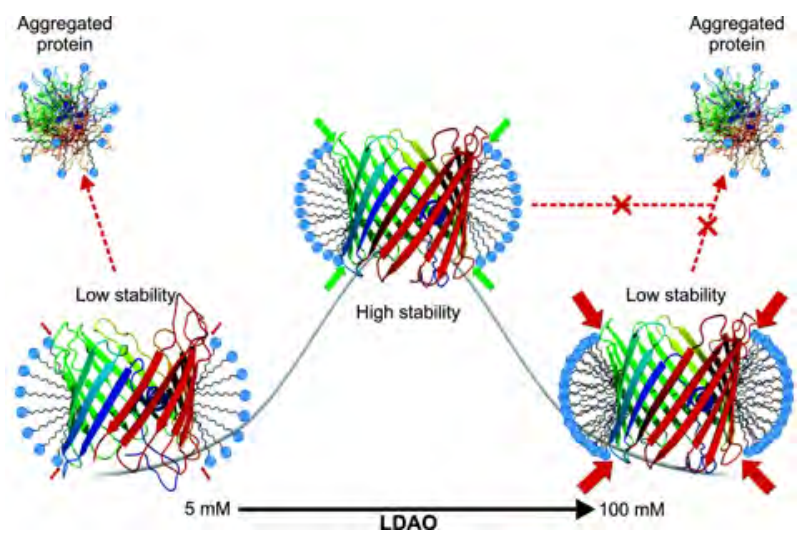

Fig. 15: Schematic illustrating the role of DPR (LDAO concentration) on the stability of hVDAC-2. Both low and high DPRs destabilize hVDAC-2, resulting in protein unfolding and is often accompanied by protein aggregation. We speculate that the hVDAC-2 barrel requires an optimal lateral pressure (represented using the green and red arrows) from the micellar assembly, in order to remain folded. Figure reproduced with permission from Maurya and Mahalakshmi (2014b) 
barrel is sensitive to the lateral pressure exerted by micelle packing and crowding of detergent molecules (Maurya and Mahalakshmi, 2013). Results in similar lines from controlled micellar systems are invaluable to understand factors modulating membrane protein stability, as well as further our understanding of proteinprotein and protein-lipid interactions in vivo.

\section{Conclusions}

Membrane proteins have intrigued and fascinated scientists with their diverse behavioural characteristics and functions. We are only beginning to comprehend rules that govern membrane protein structure and stability-factors that are key to its function and regulation within the cell. The development of membrane mimetics, and the advancement of methodology earlier available only for soluble proteins, to the study of membrane protein systems, has significantly advanced our understanding of these proteins and provided us with new avenues to examine these systems in greater detail. For instance, $\Phi$-value analysis, wherein the energetic contribution of residues to the transition state of a two-state unfolding process, has traditionally been carried out for soluble proteins. The extension of $\Phi$-value analysis to membrane protein systems has allowed scientists to map key residue types and their positional significance to the refolding pathway of transmembrane $\beta$-barrels (Huysmans et al., 2010). Similarly, the development of mixed bicelles and nanodiscs has enabled structure determination of these proteins, both in solution using NMR and in the solid state using crystallography (Durr et al., 2012). Our work on $\beta$-barrel OMPs from bacterial and human origin, has allowed us to map the contribution of the refolding milieu and key interface residues to the thermodynamic and kinetic stabilization of these membrane proteins.

Despite the progress in characterization of membrane proteins, we are considerably lagging behind, in comparison with our current knowledge of soluble proteins. The generation of sufficient quantities of protein for use in biochemical studies in vitro (Gupta et al., 2012; Huysmans et al., 2010; Moon et al., 2013; Pocanschi et al., 2013), and rapid biophysical screens of protein stability and function
(Gupta et al., 2015), would help us translate these observations to in vivo conditions. A reductionist approach to the study of specifically engineered interactions, as demonstrated earlier for aromatic interactions (Fig. 16) (Mahalakshmi et al., 2006; Makwana et al., 2013; Makwana and Mahalakshmi, 2014a; Makwana and Mahalakshmi, 2014b; Sengupta et al., 2005), can also be employed to specifically address the contribution of key interactions to the stability of membrane proteins. For example, it is known in PagP that tertiary interactions between a Trp-Tyr pair are observed in the folded state of the barrel, which is believed to give rise to a signature tertiary CD spectrum at $231 \mathrm{~nm}$, in this protein (Khan et al., 2007). Such interactions not only contribute to the scaffold stability, but also serve as excellent noninvasive probes for the study of membrane protein folding.

Using a few carefully chosen membrane proteins, different strategies to extract underlying

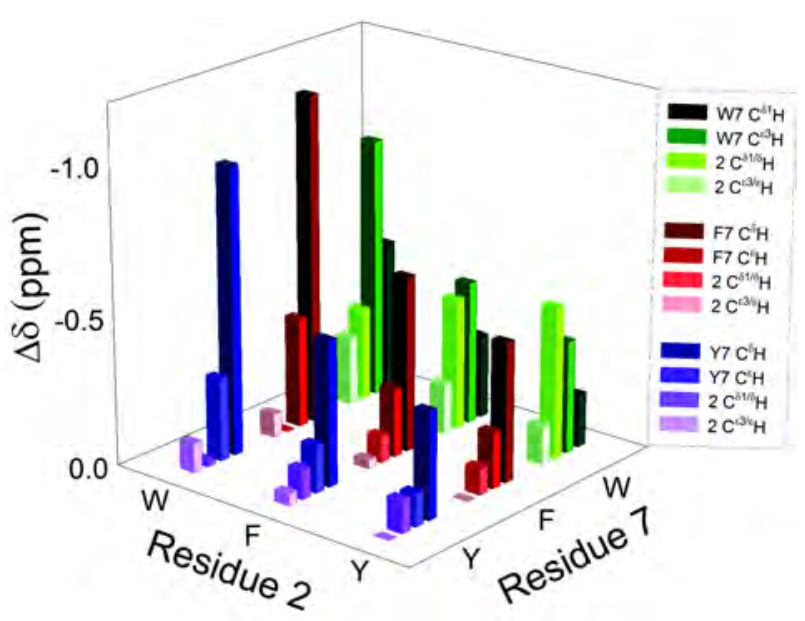

Fig. 16: Use of chemical shift differences $(\Delta \delta)$ between pairs of interacting aromatic amino acids selectively positioned on designed octapeptide hairpin secondary structure scaffolds, to assess the interaction strength of various aryl pair permutants. All peptides have been designed such that the aromatic residues, placed at positions 2 and 7 in the octapeptide, exhibit Tshaped geometries in solution. Higher $\Delta \delta$ values of the $7^{\text {th }}$ position indicate stronger T-shaped face-toedge aromatic interactions, and are generally indicative of well-folded hairpin scaffolds, as a consequence of this interacting aryl pair. Figure reproduced with permission from Makwana and Mahalakshmi (2014a) 
similarities and differences in membrane protein folding and function in vitro have been developed thus far. We are yet to understand how membrane protein complexes are stabilized both in vitro and in vivo, and deduce factors that govern the transient formation of such complexes during signalling, cellcell interaction and for executing vital functions in a resting cell. Further, several unanswered aspects of membrane protein recycling in microorganisms and higher eukaryotes require comprehensive research on real-time monitoring of these proteins, so as to obtain meaningful conclusions on how membrane proteins are processed. The fundamental question of how a membrane protein folds, and how its structure, stability and function is modulated by subtle variations in the lipid composition, is yet to be deduced at the molecular level. Since each membrane protein is unique in the way it folds and interacts with its lipid environment, it may not be currently possible to design well-defined methodologies that can be generalized

FT/LS-47/2010 and intramural funds.

\section{References}

Anbazhagan V, Vijay N, Kleinschmidt J H and Marsh D (2008) Protein-lipid interactions with Fusobacterium nucleatum major outer membrane protein FomA: spin-label EPR and polarized infrared spectroscopy Biochemistry 47 84148423

Andersen K K, Wang H and Otzen D E (2012) A kinetic analysis of the folding and unfolding of OmpA in urea and guanidinium chloride: single and parallel pathways Biochemistry $\mathbf{5 1} 8371-8383$

Andrews B T, Capraro D T, Sulkowska J I, Onuchic J N and Jennings P A (2013) Hysteresis as a Marker for Complex, Overlapping Landscapes in Proteins J Phys Chem Lett 4 180-188

Booth P J and Curnow P (2009) Folding scene investigation: membrane proteins Curr Opin Struct Biol 19 8-13

Burgess N K, Dao T P, Stanley A M and Fleming K G (2008) Beta-barrel proteins that reside in the Escherichia coli outer membrane in vivo demonstrate varied folding behavior in vitro J Biol Chem 283 26748-26758

Chattopadhyay A, Mukherjee S, Rukmini R, Rawat S S and Sudha S (1997) Ionization, partitioning, and dynamics of for all membrane proteins. However, further studies in this direction would enable us deduce principles governing membrane protein structure, function and regulation in vivo and enable successful design of structure- and mechanism-based drugs and regulatory elements to counteract pathogenesis as well as remedy debilitating neurodegenerative diseases and cancer.

\section{Acknowledgments}

A large body of the work summarized in this review has been made possible due to the efforts of graduate students, past and present research fellows and past research trainees of the R. M. laboratory. R. M. is a recipient of the Ramalingaswami Fellowship from the Department of Biotechnology (DBT), Government of India. Work in the R. M. laboratory has been funded by the DBT award numbers BT/HRD/35/02/ 25/2009 and BT/01/IYBA/2009, the Science and Engineering Research Board, Department of Science and Technology, Govt. of India award number SR/

tryptophan octyl ester: implications for membrane-bound tryptophan residues Biophys $J \mathbf{7 3}$ 839-849

Chaturvedi D and Mahalakshmi R (2013) Methionine mutations of outer membrane protein X influence structural stability and beta-barrel unfolding PLoS One 8 e79351

Chaturvedi D and Mahalakshmi R (2014) Juxtamembrane tryptophans have distinct roles in defining the OmpX barrel-micelle boundary and facilitating protein-micelle association FEBS Lett $\mathbf{5 8 8}$ 4464-4471

Curnow P and Booth P J (2007) Combined kinetic and thermodynamic analysis of alpha-helical membrane protein unfolding Proc Natl Acad Sci U S A 104 18970-18975

Durr U H, Gildenberg M and Ramamoorthy A (2012) The magic of bicelles lights up membrane protein structure Chem Rev 112 6054-6074

Faham S, Yang D, Bare E, Yohannan S, Whitelegge J P and Bowie J U (2004) Side-chain contributions to membrane protein structure and stability J Mol Biol 335 297-305

Fleming K G, Ackerman A L and Engelman D M (1997) The effect of point mutations on the free energy of transmembrane alpha-helix dimerization J Mol Biol 272 266-275

Grosse W, Psakis G, Mertins B, Reiss P, Windisch D, Brademann F, Burck J, Ulrich A, Koert U and Essen L O (2014) 
Structure-based engineering of a minimal porin reveals loopindependent channel closure Biochemistry 53 4826-4838

Gupta A, Chaturvedi D and Mahalakshmi R (2012) Modified $\mathrm{CNBr}$ cleavage protocol for efficient separation of MetSer containing OmpX-Om14 membrane protein fusion International Review of Biophysical Chemistry 3 147-156

Gupta A, Zadafiya P and Mahalakshmi R (2014) Differential Contribution of Tryptophans to the Folding and Stability of the Attachment Invasion Locus Transmembrane betaBarrel from Yersinia pestis Sci Rep 46508

Gupta A, Iyer B R, Chaturvedi D, Maurya S R and Mahalakshmi R (2015) Thermodynamic, structural and functional properties of membrane protein inclusion bodies are analogous to purified counterparts: Case study from bacteria and humans $R$ Soc Chem Adv 5 1227-1234

Hong H and Tamm L K (2004) Elastic coupling of integral membrane protein stability to lipid bilayer forces Proc Natl Acad Sci U S A 101 4065-4070

Hong H, Park S, Jimenez R H, Rinehart D and Tamm L K (2007) Role of aromatic side chains in the folding and thermodynamic stability of integral membrane proteins $J$ Am Chem Soc 129 8320-8327

Hong H, Rinehart D and Tamm L K (2013) Membrane depthdependent energetic contribution of the tryptophan side chain to the stability of integral membrane proteins Biochemistry 52 4413-4421

Huysmans G H, Radford S E, Brockwell D J and Baldwin S A (2007) The N-terminal helix is a post-assembly clamp in the bacterial outer membrane protein PagP J Mol Biol 373 529-540

Huysmans G H, Baldwin S A, Brockwell D J and Radford S E (2010) The transition state for folding of an outer membrane protein Proc Natl Acad Sci U S A 107 4099-4104

Jefferson R E, Blois T M and Bowie J U (2013) Membrane proteins can have high kinetic stability $\mathrm{J}$ Am Chem Soc 135 15183-15190

Kelkar D A and Chattopadhyay A (2006) Membrane interfacial localization of aromatic amino acids and membrane protein function J Biosci 31 297-302

Khan M A, Neale C, Michaux C, Pomes R, Prive G G, Woody R W and Bishop R E (2007) Gauging a hydrocarbon ruler by an intrinsic exciton probe Biochemistry 46 4565-4579

Killian J A and von Heijne G (2000) How proteins adapt to a membrane-water interface Trends Biochem Sci 25 429434

Kleinschmidt J H and Tamm L K (1996) Folding intermediates of a beta-barrel membrane protein. Kinetic evidence for a multi-step membrane insertion mechanism Biochemistry

\section{12993-13000}

Kleinschmidt J H, den Blaauwen T, Driessen A J and Tamm L K (1999a) Outer membrane protein A of Escherichia coli inserts and folds into lipid bilayers by a concerted mechanism Biochemistry 38 5006-5016

Kleinschmidt J H and Tamm L K (1999) Time-resolved distance determination by tryptophan fluorescence quenching: probing intermediates in membrane protein folding Biochemistry 38 4996-5005

Kleinschmidt J H, Wiener M C and Tamm L K (1999b) Outer membrane protein A of E. coli folds into detergent micelles, but not in the presence of monomeric detergent Protein Sci 8 2065-2071

Kleinschmidt J H and Tamm L K (2002) Secondary and tertiary structure formation of the beta-barrel membrane protein OmpA is synchronized and depends on membrane thickness J Mol Biol 324 319-330

Kleinschmidt J H (2006) Folding kinetics of the outer membrane proteins OmpA and FomA into phospholipid bilayers Chem Phys Lipids 141 30-47

Lella M and Mahalakshmi R (2013) Pro-Gly mediated conformational switch of mycobacteriophage D29 holin transmembrane domain I is lipid concentration driven Chem Commun 49 9594-9596

Mahalakshmi R, Raghothama S and Balaram P (2006) NMR analysis of aromatic interactions in designed peptide betahairpins J Am Chem Soc 128 1125-1138

Mahalakshmi R, Franzin C M, Choi J and Marassi F M (2007) NMR structural studies of the bacterial outer membrane protein OmpX in oriented lipid bilayer membranes Biochim Biophys Acta 1768 3216-3224

Mahalakshmi R and Marassi F M (2008) Orientation of the Escherichia coli outer membrane protein OmpX in phospholipid bilayer membranes determined by solid-State NMR Biochemistry 47 6531-6538

Makwana K M, Raghothama S and Mahalakshmi R (2013) Stabilizing effect of electrostatic vs. aromatic interactions in diproline nucleated peptide beta-hairpins Phys Chem Chem Phys 15 15321-15324

Makwana K M and Mahalakshmi R (2014a) Asymmetric contribution of aromatic interactions stems from spatial positioning of the interacting aryl pairs in beta-hairpins ChemBioChem 15 2357-2360

Makwana K M and Mahalakshmi R (2014b) Comparative analysis of cross strand aromatic-Phe interactions in designed peptide beta-hairpins Org Biomol Chem 12 20532061

Maurya S R, Chaturvedi D and Mahalakshmi R (2013) 
Modulating lipid dynamics and membrane fluidity to drive rapid folding of a transmembrane barrel Sci Rep 31989

Maurya S R and Mahalakshmi R (2013) Modulation of human mitochondrial voltage-dependent anion channel 2 (hVDAC2) structural stability by cysteine-assisted barrel-lipid interactions J Biol Chem 288 25584-25592

Maurya S R and Mahalakshmi R (2014a) Cysteine residues impact the stability and micelle interaction dynamics of the human mitochondrial beta-barrel anion channel hVDAC-2 PLoS One 9 e92183

Maurya S R and Mahalakshmi R (2014b) Influence of proteinmicelle ratios and cysteine residues on the kinetic stability and unfolding rates of human mitochondrial VDAC-2 PLoS One 9 e 87701

Moon C P and Fleming K G (2011) Side-chain hydrophobicity scale derived from transmembrane protein folding into lipid bilayers Proc Natl Acad Sci U S A 108 10174-10177

Moon C P, Kwon S and Fleming K G (2011) Overcoming hysteresis to attain reversible equilibrium folding for outer membrane phospholipase A in phospholipid bilayers $J$ Mol Biol 413 484-494

Moon C P, Zaccai N R, Fleming P J, Gessmann D and Fleming K G (2013) Membrane protein thermodynamic stability may serve as the energy sink for sorting in the periplasm Proc Natl Acad Sci U S A $1104285-4290$

Otzen D E (2002) Protein unfolding in detergents: effect of micelle structure, ionic strength, $\mathrm{pH}$, and temperature Biophys $J$ 83 2219-2230

Otzen D E and Oliveberg M (2002) Burst-phase expansion of native protein prior to global unfolding in SDS $J$ Mol Biol 315 1231-1240

Otzen D E and Andersen K K (2013) Folding of outer membrane proteins Arch Biochem Biophys 531 34-43

Parker M J and Marqusee S (1999) The cooperativity of burst phase reactions explored J Mol Biol 293 1195-1210

Plesniak L A, Mahalakshmi R, Rypien C, Yang Y, Racic J and Marassi F M (2011) Expression, refolding, and initial structural characterization of the Y. pestis Ail outer membrane protein in lipids Biochim Biophys Acta 1808 482-489

Pocanschi C L, Apell H J, Puntervoll P, Hogh B, Jensen H B, Welte W and Kleinschmidt J H (2006a) The major outer membrane protein of Fusobacterium nucleatum (FomA) folds and inserts into lipid bilayers via parallel folding pathways J Mol Biol 355 548-561

Pocanschi C L, Patel G J, Marsh D and Kleinschmidt J H (2006b) Curvature elasticity and refolding of OmpA in large unilamellar vesicles Biophys J 91 L75-77

Pocanschi C L, Popot J L and Kleinschmidt J H (2013) Folding and stability of outer membrane protein A (OmpA) from Escherichia coli in an amphipathic polymer, amphipol A835 Eur Biophys J 42 103-118

Rasmussen A, Rasmussen T, Edwards MD, Schauer D, Schumann U, Miller S and Booth I R (2007) The role of tryptophan residues in the function and stability of the mechanosensitive channel MscS from Escherichia coli Biochemistry 46 10899-10908

Sanchez-Ruiz J M (2010) Protein kinetic stability Biophys Chem $1481-15$

Sanchez K M, Gable J E, Schlamadinger D E and Kim J E (2008) Effects of tryptophan microenvironment, soluble domain, and vesicle size on the thermodynamics of membrane protein folding: lessons from the transmembrane protein OmpA Biochemistry 47 12844-12852

Sanchez K M, Kang G, Wu B and Kim J E (2011) Tryptophanlipid interactions in membrane protein folding probed by ultraviolet resonance Raman and fluorescence spectroscopy Biophys J 100 2121-2130

Sehgal P, Mogensen J E and Otzen D E (2005) Using micellar mole fractions to assess membrane protein stability in mixed micelles Biochim Biophys Acta 1716 59-68

Sengupta A, Mahalakshmi R, Shamala N and Balaram P (2005) Aromatic interactions in tryptophan-containing peptides: crystal structures of model tryptophan peptides and phenylalanine analogs J Pept Res 65 113-129

Shanmugavadivu B, Apell H J, Meins T, Zeth K and Kleinschmidt J H (2007) Correct folding of the beta-barrel of the human membrane protein VDAC requires a lipid bilayer $\mathrm{J} \mathrm{Mol}$ Biol 368 66-78

Stanley A M and Fleming K G (2008) The process of folding proteins into membranes: challenges and progress Arch Biochem Biophys 469 46-66

Sun H, Greathouse D V, Andersen O S and Koeppe R E, 2nd (2008) The preference of tryptophan for membrane interfaces: insights from $\mathrm{N}$-methylation of tryptophans in gramicidin channels J Biol Chem $28322233-22243$

Yamashita S, Lukacik P, Barnard T J, Noinaj N, Felek S, Tsang T M, Krukonis E S, Hinnebusch B J and Buchanan S K (2011) Structural insights into Ail-mediated adhesion in Yersinia pestis Structure 19 1672-1682. 